\title{
Tratamiento mediante dilatación con balón de un paciente con estenosis subaórtica por membrana: a propósito de un caso
}

\author{
Percutaneous balloon dilatation in a patient with membrane subaortic \\ stenosis: a propos of a case
}

\author{
Carlos Mariño ${ }^{1, a}$, Julio Castillejo ${ }^{2, a}$, Carlos Morales ${ }^{3, a}$, Hernán Vela $^{1}$, César Salinas ${ }^{1}$ \\ Servicio de Cardiología, Instituto Nacional de Salud del Niño, Lima, Perú. \\ ${ }^{2}$ Servicio de Anestesiologia, Hospital Guillermo Almenara, EsSalud, Lima, Perú. \\ ${ }^{3}$ Servicio de Medicina, Instituto Nacional de Salud del Niño, Lima, Perú. \\ ${ }^{a}$ Maestrando en Docencia e Investigación en Salud, Universidad Nacional Mayor de San Marcos.
}

\begin{abstract}
Resumen
La estenosis subaórtica es una lesión cardiaca poco frecuente, en el cual el tracto de salida del ventrículo izquierdo está estrechado por la presencia de un anillo fibroso o fibromuscular. Constituye 8 a $20 \%$ de todas las formas de obstrucción al tracto de salida del ventrículo izquierdo. La cirugia ha sido considerada el tratamiento de elección cuando el gradiente pico es mayor de 50 $\mathrm{mmHg} 0$ en pacientes que, con gradientes menores, presentan progresión de la insuficiencia aórtica o dilatación de los diámetros ventriculares, disfunción ventricular o síntomas. Presentamos el caso de un paciente menor de edad, portador de estenosis subaórtica por membrana, con síntomas de insuficiencia cardiaca y función ventricular izquierda muy comprometida, en la que se realizó el tratamiento percutáneo, alternativo al quirúrgico, con resultado exitoso. Constituye el primer caso reportado en el país de tratamiento percutáneo con balón y con evolución favorable.
\end{abstract}

Palabras clave: Estenosis subaórtica, tratamiento percutáneo, dilatación con balón.

\section{Abstract}

Discrete subaortic stenosis is a rare cardiac lesion with left ventricle outflow narrowed by the presence of a muscular or fibrous ring. It accounts for $8-20 \%$ of all forms of left ventricle outflow tract obstruction. Surgery is considered the treatment of choice when the peak gradient is over $50 \mathrm{mmHg}$ or in patients with lower gradients and either progression of aortic regurgitation, dilated ventricular diameters, left ventricular dysfunction or symptoms. We report the case of a child with membrane subaortic stenosis and symptoms of heart failure and compromised left ventricular function, in whom percutaneous balloon dilatation was performed as an alternative to surgery. This is the first case reported in the country with successful outcome.

Keywords: Discrete subaortic stenosis, percutaneous treatment, balloon dilation.

\section{An Fac med. 2013;74(4):327-30}

\section{INTRODUCCIÓN}

La aparición de una membrana fina circunferencial o en forma de media luna en el tracto de salida ventricular izquierdo constituye la forma más frecuente y leve del espectro de la estenosis subaórtica ${ }^{(1)}$. El grado de severidad hemodinámica es variable, influyendo en la posible incidencia de complicaciones (insuficiencia aórtica, endocarditis o enfermedad muscular obstructiva). Por todo esto, la estenosis subaórtica de membrana es considerada una enfermedad potencialmente progresiva ${ }^{(2)}$.

La cirugía ha sido considerada el tratamiento de elección cuando el gradiente pico es mayor de $50 \mathrm{mmHg}$ o en pacientes que, con gradientes menores, presentan progresión de la insuficiencia aortica o de los diámetros ventriculares, disfunción ventricular o síntomas.
Pese a ser un tratamiento seguro y efectivo, especialmente en casos provocados por membrana fina, no está exenta de complicaciones en el seguimiento o de recurrencias de la enfermedad, que pudieran implicar la necesidad de una nueva intervención ${ }^{(3)}$.

Se presenta el caso de un paciente portador de estenosis subaórtica por membrana, cuyo tratamiento usualmente quirúrgico fue realizado en el 
Laboratorio de Hemodinámica del Instituto Nacional de Salud del Niño mediante la técnica alternativa percutánea con balón, constituyendo el primer procedimiento exitoso comunicado en el país. Ello implicaría un cambio muy importante en el abordaje del manejo no quirúrgico de esta enfermedad en nuestro centro hospitalario.

\section{CASO CLÍNICO}

Paciente varón de 11 años de edad, procedente de la ciudad del Cuzco. Acudió al Servicio de Cardiología del Instituto Nacional de Salud del Niño con cuadro clínico de 6 meses de evolución, caracterizado por disnea a medianos esfuerzos (al caminar menos de 100 metros) y dolor torácico opresivo.

$\mathrm{Al}$ ingreso, el paciente tenía un peso de $26 \mathrm{~kg}$, presión arterial de 85/60, frecuencia cardiaca de $80 \mathrm{x}$ minuto y saturación de oxígeno de $96 \%$. El paciente no mostraba facies característica, estaba hidratado y adelgazado.

$\mathrm{Al}$ examen, la piel era tibia, elástica, pálida, sin equimosis ni petequias. El tejido celular subcutáneo estaba disminuido, no había edemas. No se palpó adenomegalias en las regiones cervical, axilar o inguinal.

En la auscultación pulmonar se encontró el murmullo disminuido en ambos hemitórax. En el aparato cardiovascular, los ruidos cardiacos fueron rítmicos, el segundo ruido cardiaco disminuido en intensidad, y presencia de soplo sistólico de grado 3/6 (escala de Levine), irradiado al cuello. En las extremidades, los pulsos periféricos tenían poca amplitud e intensidad. El abdomen era blando, depresible, con ruidos hidroaéreos presentes; había visceromegalia importante, hígado a 4 $\mathrm{cm}$ debajo del reborde costal derecho. No se encontró compromiso articular, esquelético o muscular. El examen neurológico fue normal.

Entre los exámenes auxiliares, en el hemograma había leucocitosis de 12 700, sin desviación izquierda; el hematocrito era 39\%, la hemoglobina 12,7, las plaquetas 204000 . El reactante de fase aguda PCR fue 0,16. El EKG mostró ritmo sinusal, hipertrofia ventricular izquierda y patrón de sobrecarga de volumen. Entre los exámenes de imagen, en las placas de rayos X se observó cardiomegalia, con un índice cardiotorácico de 0,60 , crecimiento de ventrículo izquierdo y signos sugestivos de hipertensión venocapilar.

El ecocardiograma transtorácico (ETT) tuvo los siguientes hallazgos: cámaras izquierdas dilatadas. Hipertrofia del ventrículo izquierdo (VI), función sistólica del VI severamente disminuida (fracción de eyección (FE): 29\%), regurgitación mitral leve. Aorta trivalva, con anillo de $16 \mathrm{~mm}$. Estenosis subaórtica por membrana, con gradiente de $148 \mathrm{mmHg}$ (figura 1).

Fue necesario complementar la evaluación de la enfermedad con un ecocardiograma transesofágico (ETE), que encontró una membrana subaórtica que producía estenosis severa con gradiente de $90 \mathrm{mmHg}$, y gradiente medio de $60 \mathrm{mmHg}$. Hipertrofia excéntrica del VI de grado severo. Hipocinesia difusa a predominio del segmento antero septo-apical. Crecimiento de aurícula izquierda. Compromiso severo de la función del VI (FE: 30\%). Disfunción diastólica restrictiva tipo III. Velocidades miocárdicas severamente disminuidas. Aumento severo de la presión de fin de diástole.

Durante la hospitalización el paciente cursó con disnea progresiva a mínimos esfuerzos, que requirió de manejo intensivo de la insuficiencia cardiaca, por lo que se consideró inicialmente el tratamiento quirúrgico como primera elección. Sin embargo, debido a dificultades en la disponibilidad de sala de operaciones y ante la necesidad de un tratamiento de urgencia, se optó por el tratamiento alternativo percutáneo.

Previo consentimiento informado, se realizó el tratamiento percutáneo con balón en el Laboratorio de Hemodinámica del INSN, mediante cateterismo cardiaco izquierdo.

El paciente fue intubado, bajo anestesia general. Se abordó la arteria femoral derecha y se avanzó un catéter JR para registrar una diferencia de presiones entre aorta y ventrículo izquierdo de $105 \mathrm{mmHg}$. Luego, sobre una guía de intercambio de alto soporte situada en la cavidad ventricular izquierda se avanzó el catéter balón. Se seleccionó el tamaño del balón en base al diámetro angiográfico del anillo aórtico, en

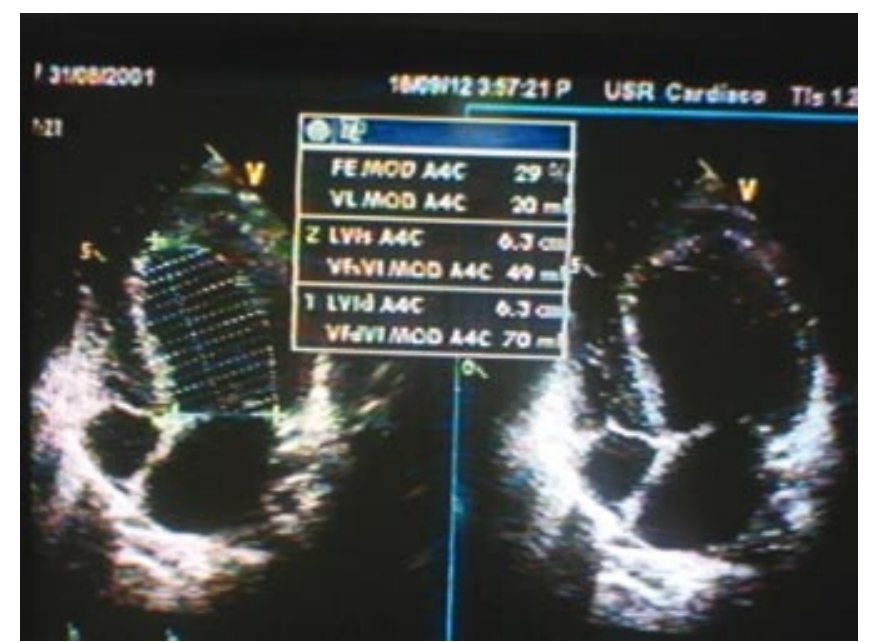

Figura 1. Ecografía transtorácica. Vista de cuatro cámaras: dilatación severa de cámaras cardiacas izquierdas, con disminución importante de la función sistólica del ventrículo izquierdo (FE: 29\%). 


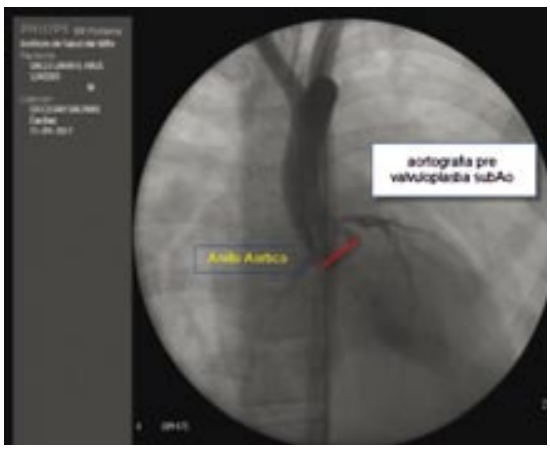

Figura 2. Aortografía predilatación. Medición del anillo aórtico=16 $\mathrm{mm}$ y selección de balón de valvuloplastia.

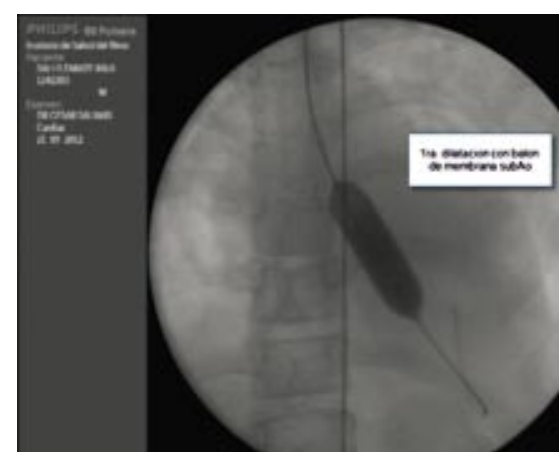

Figura 3. Dilatación aórtica. Primera valvuloplastia subaórtica con balón de $14 \mathrm{~mm}$ (ancho) X $4 \mathrm{~cm}$ (largo).

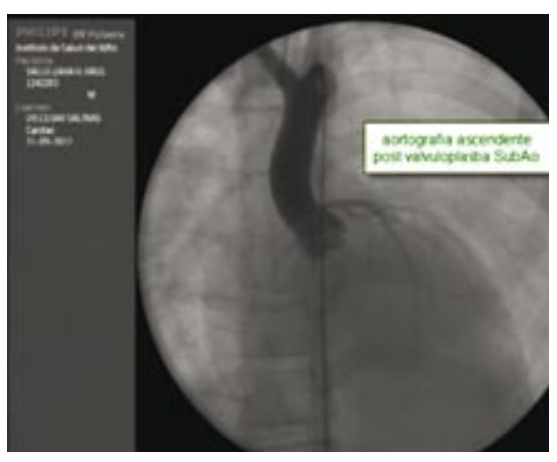

Figura 4. Aortografía posdilatación con balón. Control angiográfico (aortografía ascendente) para valorar insuficiencia valvular aórtica residual. nuestro caso, de $16 \mathrm{~mm}$ (figura 2). Se usó inicialmente un balón de 14 x 4 y luego otro de $16 \times 4$, con insuflados repetidos hasta conseguir la desaparición completa de la muesca provocada por la membrana (figura 3).

No fue necesario usar durante el inflado del balón algún método que disminuyera la contractilidad cardíaca y aportara una mayor estabilidad (por ejemplo, estimulación cardiaca con marcapaso). La gradiente final VI-aorta posdilatación con balón fue $24 \mathrm{mmHg}$, y en la angiografía de control inmediata se registró mínima insuficiencia aórtica (figura 4). No hubo complicación inmediata relacionada al procedimiento.

La evolución clínica fue favorable, y el control ecocardiográfico transtorácico a las 24 horas evidenció mejoría importante de la contractilidad miocárdica del VI con FE: 74\% y gradiente residual de $60 \mathrm{mmHg}$. Hipertrofia leve del ventrículo izquierdo e insuficiencia mitral leve. En los días posteriores mantuvo una evolución satisfactoria, con remisión gradual de la sintomatología de insuficiencia cardiaca, por lo cual fue dado de alta a la semana, con medicación farmacológica.

\section{DISCUSIÓN}

La estenosis subaórtica (ESAo) presenta dos tipos anatomopatológicos: una estenosis de tramo corto debida a mem- brana (anillo fibroso o fibromuscular) y otro tipo en el que un largo segmento por debajo de la aorta estaría estrecho (más de 1/3 del diámetro de la aorta), llamada también tuneliforme, que es mucho más rara ${ }^{(4,5)}$. Para el presente reporte de caso nosotros describimos el tratamiento percutáneo de un paciente con estenosis subaórtica por membrana.

La ESAo se puede presentar de forma aislada o con otras anomalías cardiacas $(50 \%$ de los pacientes), siendo la más común la comunicación intraventricular (CIV). La estenosis puede aparecer en estos pacientes incluso después de la corrección de su cardiopatía. Por otro lado, muchos pacientes (hasta $50 \%$ ) presentan insuficiencia aórtica; el mecanismo de producción de esta sería el daño de la válvula aórtica ocasionado por el jet sistólico anormal ${ }^{(6,7)}$.

En general, los niños no tienen síntomas debido a que la ESAo es evolutiva y es extraño encontrar en la infancia gradientes severos. Lo más frecuente es que se valore al niño por un soplo o que se descubra la membrana subaórtica al realizar una ecocardiografía al valorar alguna cardiopatía, como por ejemplo una CIV. Debido a que los síntomas guardan relación directa con el grado de obstrucción, la sintomatología se presenta cuando este es moderado a severo. El paciente puede presentar disnea de grado variable, así como mareos, dolor precordial y síncope. La le- sión severa suele ocurrir generalmente en la edad adulta, con fallecimiento del paciente hacia la cuarta y quinta década de la vida si la obstrucción no es tratada quirúrgicamente ${ }^{(8)}$. Sin embargo, en nuestro paciente la presentación de severidad fue más precoz, manifestándose sintomatología de insuficiencia cardiaca en un menor de 11 años, con compromiso severo de la función sistólica del VI, que además fue tratado por método intervencionista.

El tratamiento percutáneo de la estenosis subaórtica por membrana es una alternativa cada vez más utilizada por diversos grupos intervencionistas ${ }^{(9,10)}$, con resultados prometedores. En nuestro país, habitualmente se realiza la reparación quirúrgica que, pese a ser el tratamiento estándar, seguro y efectivo, no está exenta de complicaciones en el seguimiento o de recurrencias de la enfermedad, que pudieran implicar la necesidad de una nueva intervención ${ }^{(11,12)}$. El presente caso de tratamiento con balón es el primero en ser realizado en nuestra institución y el primero en ser reportado para publicación con resultado exitoso. Puede constituir una alternativa muy valiosa en nuestro medio para el manejo de la estenosis subaórtica, con muchas ventajas para nuestros pacientes, pues es menos invasiva respecto a la cirugía, evita la esternotomía y el uso de circulación extracorpórea. Además, al no requerir de sala de operaciones ni del personal de cirugía cardiaca, optimi- 
za el recurso humano para realización de otro tipo de cirugías cardiacas más complejas, con alta demanda en nuestra institución.

Para el resultado exitoso, debe realizarse previamente una evaluación y selección adecuada del paciente, con diagnóstico preciso (idealmente un ecocardiograma transesofágico (ETE) previo), a fin de visualizar la obstrucción subaórtica y diferenciarla del tipo túnel (o anillo fibromuscular), valorar el grosor y distancia de la membrana a la válvula aórtica, así como la evaluación de la insuficiencia aórtica ${ }^{(13)}$.

El beneficio es aún mayor respecto a la cirugía en los pacientes con función sistólica comprometida y dilatación del VI, como en nuestro paciente, en el cual el riesgo quirúrgico perioperatorio era muy alto.

Se realizó la ecocardiografía transtorácica y luego ETE, que determinaron la presencia de una membrana subaórtica delgada que producía una obstrucción del tracto de salida del ventrículo izquierdo (TSVI) con gradiente $>70$ $\mathrm{mmHg}$, en ausencia de insuficiencia aortica significativa. Además, se evidenció dilatación importante del ventrículo izquierdo con disminución severa de la FE: 42\%. Clínicamente, el paciente se encontraba en insuficiencia cardiaca congestiva (ICC) III-IV. La alternativa quirúrgica fue considerada de mayor riesgo quirúrgico respecto al intervencionismo, por lo cual se optó por esta última.

Previa firma de consentimiento informado, se programó al paciente para terapia percutánea de dilatación con balón. Bajo anestesia general, intubado, se utilizó la técnica habitual descrita para estos casos, acceso por arteria femoral e introductor en vena femoral (alternativo para uso de catéter marpacaso). La gradiente TSVI-Ao fue 105
$\mathrm{mmHg}$, realizándose aortografía para valorar la insuficiencia aórtica y medir el anillo aórtico $(16 \mathrm{~mm})$. Se escogió un balón relación 1:1. Luego de tres dilataciones secuenciales, la gradiente disminuyó hasta $27 \mathrm{mmHg}$.

Nuestro resultado inmediato fue favorable. Mínima insuficiencia aórtica. No se registró complicaciones mayores inmediatas. A las 24 horas, el ecocardiograma evidenció mejoría de la función sistólica del ventrículo izquierdo; y a la semana, importante disminución de las cámaras ventriculares izquierdas, con recuperación de la fracción de eyección.

Para la decisión de intervención percutánea, en nuestro caso se consideró los siguientes criterios ${ }^{(13)}$ : presencia de membrana delgada y fija $<3 \mathrm{~mm}$ en grosor, existencia a nivel subaórtico de una cámara de presión demostrada hemodinámicamente y ausencia de insuficiencia aórtica significativa.

Aún cuando se presenta un solo caso, es importante considerarlo en nuestro grupo como el inicio de un manejo alternativo a la cirugía cardiaca, con un abordaje inicial menos agresivo. Los próximos casos seleccionados que cumplan los criterios de selección permitirán establecer el grado de eficacia y de seguridad del procedimiento intervencionista de la estenosis subaórtica por membrana en nuestra institución, así como comparar las complicaciones a mediano y largo plazo frente al grupo quirúrgico.

\section{REFERENCIAS BIBLIOGRÁFICAS}

1. Darcin OT, Yagdi T, Atay Y, Egnin C, Levent E, Buket $\mathrm{S}$, Alayunt E. Discrete subaortic stenosis: surgical outcomes and follow-up results. Tex Heart Inst J. 2003;30(4):286-92.

2. Stassano $\mathrm{P}$, Di Tommaso L, Contaldo A, Monaco M, Mottola M, Musumeci A, Coronella G, Spampinato N. Discrete subaortic stenosis: long-term prognosis on the progression of the obstruction and of the aortic insufficiency. Thorac Cardiovasc Surg. 2005;53(1):23-7.

3. Garcia D, Suarez de Lezo J, Segura JM, Alvarez M, Romero M, Ojeda S, et al. Resultados a largo plazo del tratamiento mediante dilatación con balón en pacientes con estenosis subaórtica de membrana: un estudio de 24 años. Cardiocore. 2012;47(1):16-9.

4. Kitchiner D, Jackson M, Malaiya, Walsh K, Peart I, Arnold R. Incidence and prognosis of obstruction of the left ventricular outflow tract in Liverpool (1960-91): a study of 313 patients. Br Heart. 1994;71(6):588-95.

5. Ruzmetov M, Vijay P, Rodefeld MD, Turrentine MW, Brown JW. Long-term results of surgical repair in patients with congenital subaortic stenosis. Interact Cardiovasc Thorac Surg. 2006;5(3):227-33.

6. Fyler D. Anomalias de la salida aórtica. En: Fyler D, editor. Nadas Cardiología Pediátrica. $1^{\text {a }}$ edición. Madrid: Mosby, 1994:504-8.

7. Attie F, Ovseyevitz J, Buendia A, Rossi RL. Estenosis aórtica. En: Sánchez $P$, editor. Cardiología Pediátrica. Clínica y Cirugia. Barcelona: Salvat, 1986:652-68.

8. Erentug V, Bozbuga N, Kirali K, Goksedef D, Akinci E, Isik O, et al. Surgical treatment of subaortic obstruction in adolescent and adults: long-term follow-up. J Card Surg. 2005;20(1):16-21.

9. Sharma S, Bhagwat AR, Loya YS. Transluminal balloon dilatation for discrete subaortic stenosis in adults and children: early and intermediate results. J Interv Cardiol. 1991;4(2):105-9.

10. Lababidi Z, Weinhaus L, Stoeckle H Jr, Walls JT. Transluminal ballon dilatation for discrete subaortic stenosis. Am J Cardiol. 1987;59(5):423-5.

11. van Son JA, Schaff HV,Danielson GK, Hagler DJ, Puga FJ. Surgical treatment of discrete and tunnel subaortic stenosis. Late survival and risk of reoperation.Circulation. 1993;88(5 Pt 2):II 159-69.

12. Gersony WM. Natural history of discrete subvalvar aortic stenosis: management implications. J Am Coll Cardiol. 2001;38(3):843-5.

13. Shrivastava S, Dev V, Bahl VK, Saxena A. Echocardiographic determinants of outcome after percutaneous transluminal balloon dilatation of discrete subaortic stenosis. Am Heart J. 1991;122(5):1323-6.

Artículo recibido el 31 de marzo de 2013 y aceptado para publicación el 7 de abril de 2013.

Conflicto de intereses:

Los autores declaran no existir conflicto de intereses.

Correspondencia:

Carlos Mariño Vigo

Cardiólogo Intervencionista

Servicio de Cardiología, Instituto Nacional de Salud del Niño

Correo electrónico: carmarvi@hotmail.com

Celular: 997940964 